\title{
Error-tolerant Tree Matching
}

\author{
Kemal Oflazer \\ Department of Computer Engineering and Information Science, \\ Bilkent University, Ankara, TR-06533, Turkey \\ ko@cs.bilkent . edu.tr
}

\begin{abstract}
This paper presents an efficient algorithm for retrieving from a database of trees, all trees that match a given query tree approximately, that is, within a certain crror tolerance. It has natural language processing applications in searching for matches in example-based translation systems, and retrieval from lexical databases containing cntries of complex feature structures. The algorithm has been implemented on SparcStations, and for large randomly generated synthetic tree databases (some having tens of thousands of trees) it can associatively search for trees with a small error, in a matter of tenths of a second to few seconds.
\end{abstract}

\section{Introduction}

Recont approaches in machine translation known as example-based translation rely on scarching a database of previous translations of sentences or fragments of sentences, and composing a translation from the translations of any matching exarnples (Sato and Nagao, 1990; Nirenburg, Beale and Domasnhev, 1994). 'The cxample database may consist of paired text fragments, or trees as in Sato and Nagao (1990). Most often, exact matches for new sentences or fragments will not be in the database, and one has to consider exarnples that are "similar" to the sentence or fragment in question. This involves associatively searching through the database, for trees that are "close" to the query tree. This paper addresses the computational problem of retrieving trees that are close to a given query tree in terms of a certain distance metric.

'I'he paper first presents the approximate tree matching problem in an abstract setting and prescnts an algorithm for approximate associative tree matching. 'The algorithm relies on linearizing the trees and then representing the complete database of trees as a trie structure which can be efficiently searched. The problem then reduces to sequence correction problem akin to standard spelling correction problem. The trie is then used with an approximate finite state recognition algorithm close to a query tree. Following some experimental results from a number of synthetic tree databases, the paper cnds with conclusions.

\section{Approximate Tree Matching}

In this paper we consider the problem of searching in a database of trees, all trees that are "close" to a given query tree, where closeness is defined in terms of an error metric. The trees that we consider have labeled terminal and non-terminal nodes. We assume that all immediate children of a given node have unique labels, and that a total ordering on these labels is defined. We consider two trees close if we can

- add/delete a small number of leaves to/from one of the trees, and/or

- change the label of a small number of leaves in one of the trees

to get the second trec. A pair of such "close" trees is depicted in Figure 1.

\subsection{Linearization of trees}

Beforc proceeding any further we would like to define the terminology wo will be using in the following sections: We identify each leaf node in a trce with an ordered vertex list $\left(v_{0}, v_{1}, v_{2}, \cdots, v_{d}\right)$ where each $v_{i}$ is the label of a vertex from the root $v_{0}$ to the leaf $v_{d}$ at depth $d$, and for $i>0, v_{i}$ is the parent of $v_{i+1}$. A tree with $n$ leaves is represented by a vertex list sequence $V L S=V_{1}, V_{2}, \cdots, V_{n}$ where each $V_{j}=v_{0}^{j}, v_{1}^{j}, v_{2}^{j}, v_{3}^{j}, \cdots, v_{d_{j}}^{j}$ corresponds to a vertex list for a leaf at level $d_{j}$. This sequence is constructed by taking into account the total order on the labels at every level, that is, $V_{i}$ is lexicographically less than $V_{i+1}$, based on the total ordering of the vertex labels. For instance, the first tree in Figure 1 would be represented by the vertex list sequence: 

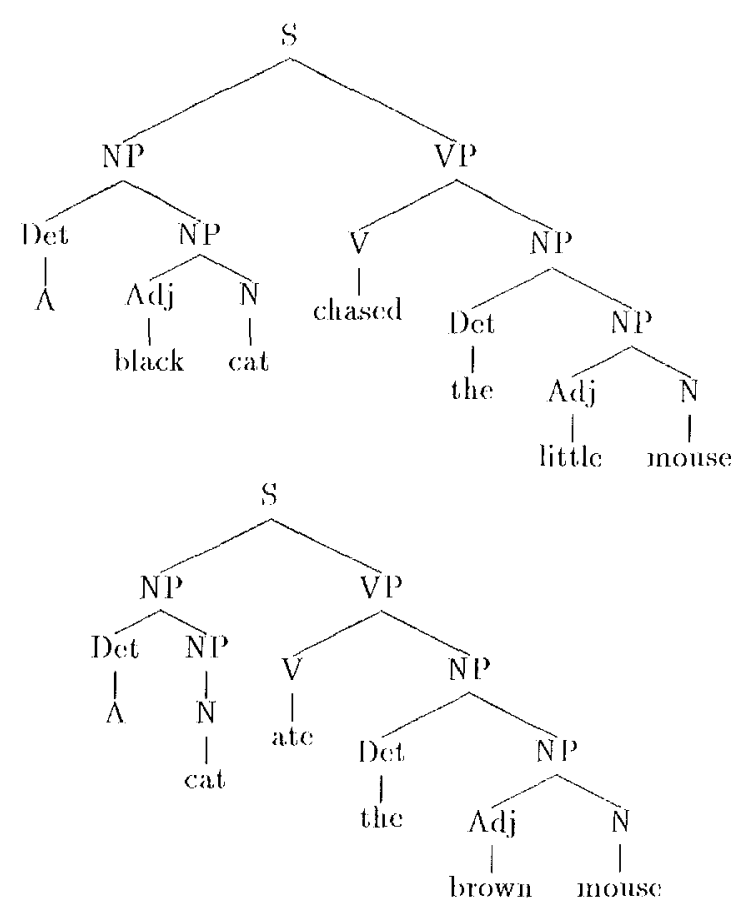

Figure 1: "Trees that are "close" to each other.

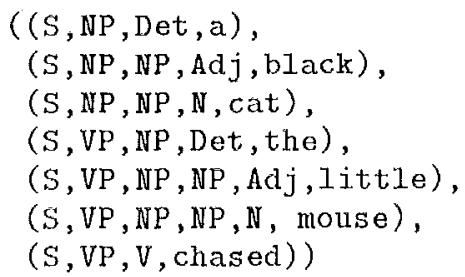

assuming the normal lexicographic ordering on node names.

\subsection{Distance between two trees}

Wo define the distanco between two trees according to the structural differences or differences in leaf labels. We consider an extra or a missing leaf as a structural change. If, however, both trees have a loaves whose vertex listis match in all but the last (leaf vertex) label, we consider this as a difference in teaf labels. For instance, in Pigure 2 , there is cxtra leaf in tree (b) in comparison to the troe in (a), while treo (c) has a leal' label differ ence. Wo associate the following costs associated wilth these differences:

- If both trees have a loal whose vertex list matches in all but the last (leaf vertex) label, wo assign a label difforence crror of $C$.

- Ir a certain leaf is missing in one of the trees but exists in the other one, then we assign a cost $s$ for this a structural difference.

We currently treat all structural or leat label differcuces as incurring a cost that, is independent of the tree kevol at which the difference takes place.<smiles>[Y]CC(C)(C)CCC</smiles>

(a)<smiles>[Y]C=C(C)C([3H])(C)C</smiles>

(b)

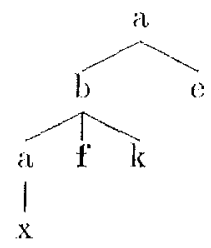

(c)

Figure 2: Structural and leaf labol differences be tween trees.

If, however, differences that are closer to the root of the tree are considered to be more serions thim differences further away from the root, it is possible to modify the formulation to take this into account.

\subsection{Converting a set of trees into a trie}

A tree dalabase $D$ consists of a set of trees $T_{1}^{1}, T_{2}, \cdots, T_{k}^{\prime}$, each $T_{i}$ being a vertex list sequenee for a tree. Once we convert all the trees to a linear form, we have a set of vertex list sequences. Wo can convert this set into a tric datia structure. 'I'his trie will compress any possible redundancies in the prefixes of the vertex list sequences to achieve a certain compaction which lielps during searching. ${ }^{1}$

For instance, the three tress in Figure 2 can be represented as a tric as shown in ligure 3. The edge labels along the path to a leaf when concitconated in order gives the vertex list so quence lor a tree, e.g., $((a, b, a, x),(a, b, c)$, $(a, b, k),(a, e))$ represents the tree (a) in Figure 2 .

\footnotetext{
${ }^{1}$ Note that it is possible to obtain more space reduction by also stharing any common postfixes of the vertex label sequences using a directed acyclic graph representation and not a tric, but this does not improve the execution time.
} 


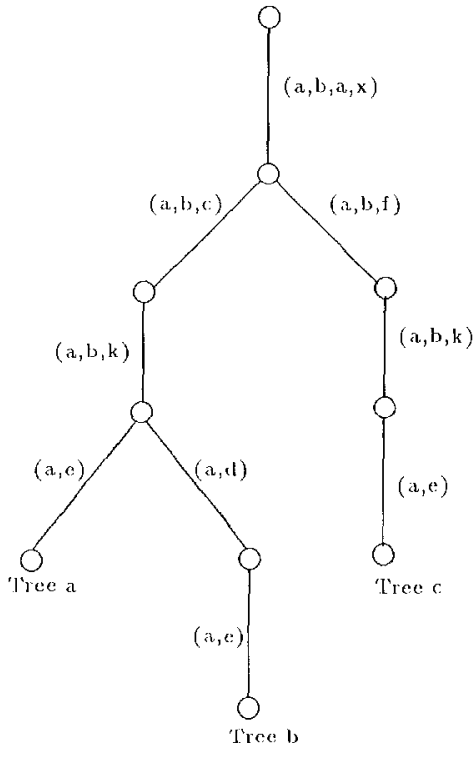

Figure 3: 'Trie representation of the 3 trees in F'igure 2

\subsection{Error-tolerant matching in the trie}

Our concern in this work is not the exact match of trees but rather approximate match. Given the vertex list sequenco for a query tree, cxact match over the trie can be performed using the stanlard techniques by following the edge labeled with next vertex list until a leaf in the trie is reached, and the query vertex label sequence is exhausted. For approximate tree matching, we use the errortolerant approximate finite-state recognition algorithm (Oflazer, 1996), which finds all strings within a given error threshold of some string in the regular set accepted by the underlying finitestate acceptor. An adaptation of this algorithm will be briefly summarized herc.

Error-tolerant matching of vertex list scquences requires an error metric for measuring how much two such sequences deviate from each other. The distance between two sequences measures the minimum number of insertions, deletions and leaf label changes that are necessary to convert one tree into another. It should be noted that this is different from the error metric defined by (Wang et al., 1994).

Let $Z=Z_{1}, Z_{2}, \ldots, Z_{p}$, denote a generic vertex list sequence of $p$ vertex lists. $Z[j]$ denotes the initial subsequence of $Z$ up to and including the $j^{\text {th }}$ leaf labcl. We will use $X$ (of length $m$ ) to denote the query vertex list sequence, and $Y$ (of length $n$ ) to denote the scquence that is a (possibly partial) candidate vertex list sequence (from the database of trces).

Given two vertex list sequences $X$ and $Y$, the distance, dist $(X[m], Y[n])$, computed according to the recurrence below, gives the minimum number of leaf insertions, deletions or leaf label changes necessary to change one trec to the other.

$$
\begin{aligned}
\operatorname{dist}(X[m], Y[n])= & \operatorname{dist}(X[m-1], Y[n-1]) \\
& \text { if } x_{m}=y_{n} \\
& (\text { last vertex lists are same }) \\
= & \operatorname{dist}(X[m-1], Y[n-1])+C \\
& \text { if } x_{m} \text { and } y_{n} \\
& \text { differ only at the } \\
& \text { leaf label } \\
= & \operatorname{dist}(X[m-1], Y[n])+S \\
& \text { if } y_{n}<x_{m}(\text { lexicographically }) \\
& X \text { is missing leaf } y_{n} . \\
= & \text { dist }(X[m], Y[n-1])+S \\
& \text { if } x_{m}<y_{n}(\text { lexicographically }) \\
& X \text { has an cxtra loaf } x_{m} . \\
& \text { Boundary Conditions } \\
\operatorname{dist}(X[0], Y[n])= & n \cdot S \\
\operatorname{dist}(X[m], Y[0])= & m \cdot S
\end{aligned}
$$

For a trec database $D$ and a distance threshold $t>0$, we considej: a query tree represented by a vertex list sequence $X[\mathrm{~m}]$ (not in the database) to match the database with an error of $t$, if the set

$$
C=\{Y[n] \mid Y[n] \in D \text { and } \operatorname{dist} X[m], Y[n]) \leq t\}
$$

is not cmply.

\subsection{An algorithm for approximate treo matching}

Standard searching with a tric corresponds to traversing a patih starting from the start node (of the tric), to one of the leal nodes (of the tric), so that the concatenation of the labels on the ares along this path matches the input vertex list sequence. For error-tolerant matching, one nceds to find all paths from the start node to one of the final nodes, so that when the labels on the edges along a path are concatenated, the resulting vertex list sequence is within a given distance threshold $t$, of the query vertex list sequence.

This scarch has to be very fast if approximate matching is to be of any practical use. 'I'his means that paths in the trie that can load to no solutions have to be pruned so that the search can be limited to a very small percentage of the search space. We need to make sure that any candidate (prefix) vertex list sequence that is gencrated as the scarch is being performed, does not deviate from cortain initial subsequences of the query sequence by more than the allowed threshold. 'Jo detect such cases, wo use the notion of a cut-off distance. The cut-off distance measures the minimum distance between an initial subsequence of the query 
sequence sequence, and the (possibly partial) candidate sequence. Iet $Y$ be a partial candidate sequence whose length is $n$, and let $X$ be the query sequence of length $m$. lot $l=\min (1, n-\lfloor t / M\rfloor)$ and $u=\max (m, n+\lceil l / M\rceil)$ where $M$ is the cost of insertions and deletions. 'The cut-off distance cutdist $(X[m], Y[n])$ is defined as

$$
\operatorname{cutdist}(X[m], Y[n])=\min _{1 \leq i \leq u} \operatorname{dist}(X[i], Y[n]) \text {. }
$$

Note hat except at the boundaries, the initial subsequences of the query sequence $X$ considered arc of length $n-\lfloor t / M\rfloor$ to length $n+[t / M\rceil$. Any initial subseruence of $X$ shorter than $/$ noeds more han $\lfloor l / M\rfloor$ lnaf node insertions, and any initial sulusting of $X$ longer than $u$ requires more than $\dashv\lceil l / M\rceil$ leaf node deletions, to at least cqual $Y$ in length, violating the distance constraint.

Given a vertex list sequenes $X$ (corresponding to a (uery tree), a partial candidate sequence $Y$ is generated by successively concatenating labels along the ares as transitions are made, starting with the start state. Whenever we extend $Y$ going along the tris, we check if the cut-ofl distance of $X$ and the partial $Y$ is within the bound specified by the threshold $l$. If the cuteoll distance gocs beyond the threshold, the last edge is backed off to the souree node (in parallel with the shortening of $Y$ ) and some other edge is tried. Backl, racking is recursively applied when the seatch can not be continued from that node. If, during the construction of $Y$, a terminal node (which may or may not be a leaf of the trie) is reached without violating the cutofl distance constraint, and $\operatorname{disl}(X[n n], Y[n]) \leq l$ at that point, then $Y$ is a tree in the database that matches the imput query sequence. 2

Denoting the nodes of the tric by subseripted $q$ 's (4o being the initial node (e.g., top node in l'igure 3) and the labels of the edges by $V$, and denoting by $\delta\left(q_{i}, V\right)$ the node in the tric that one can seach from node $q_{i}$ with edge label $V$ (denoting a vertex list.), wo present, in Figure 1, the algorithm for generating all $Y$ 's by a (slightly modified) depthCirst probing of the trie. 'Lhe crucial point in this algorithm is that the coti-olf distance computation can be performed very officiently by maindaining a matrix $/ I$ which is an $m$ by $n$ matrix with olcment $l(i, j)=\operatorname{dist}(X[i], Y[j])$ (Dn and Chang, 1992). Wo can note that the computation of the element $\Pi(i+1, j+1)$ recursively depends on only $\|(i, j)\|,(i, j+1), \Pi(i+1, j)$ from the earlier dofintion of the edit distance (see ligure 5.) D)uring the depth first scatreh of the trie, entries in column n. of the matrix $I /$ have to be (re)computed, only when the candidate string is of length $n$. During backtracking, the ontries for the last column are

\footnotetext{
${ }^{2}$ Note that we have to do this check since we may come to other irrelevant terminal nodes during the search.
}

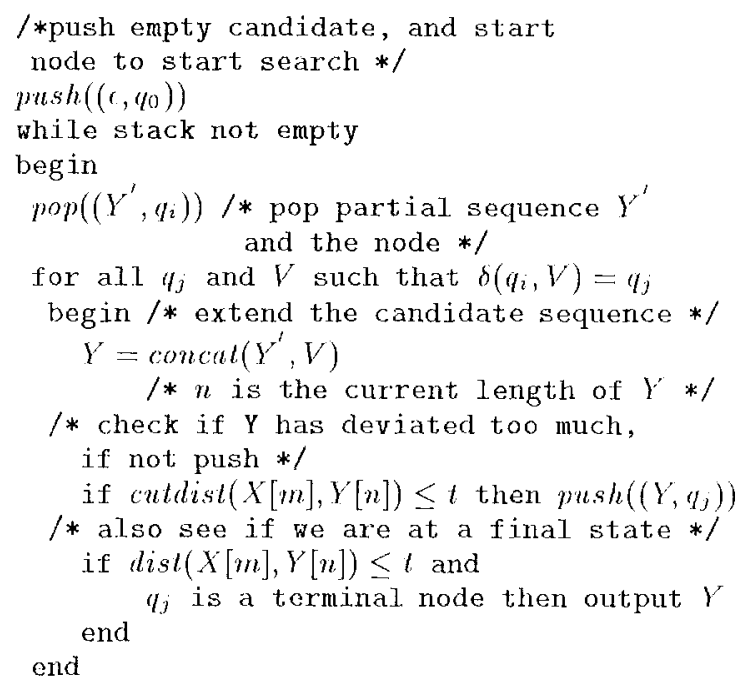

Pigure 4: Algorithm for error-tolerant recognition of vertex list sequences

$$
\left(\begin{array}{ccccc}
\cdots & \ldots & \ldots & \ldots & \ldots \\
\vdots & \vdots & \vdots & \vdots & \vdots \\
\ldots & \ldots & \ldots & \ldots & \ldots \\
\ldots & \ldots & \Pi I(i, j) & \Pi(i, j+1) & \ldots \\
\ldots & \ldots & I I(i+1, j) & I I(i+1, j+1) & \ldots \\
\vdots & \vdots & \vdots & \vdots & \vdots \\
\ldots & \ldots & \ldots & \ldots & \ldots
\end{array}\right)
$$

Figure 5: Computation of the elements of the $I 1$ matrix.

discarded, but the entries in prior colnums are still valid. 'Thus all cotries required by $H(i+1, j+1)$, except $H(i, j+1)$, are already avaliable in the matrix in columus $i-1$ and $i$. The computation of cutdist $(X[m], Y[n])$ involves a loop in which the minimum is computed. 'This loop (indexing along column $j+1$ ) compules $I I(i, j+1)$ before it is needed for the computation of $I I(i+1, j+1)$.

\section{$3 \quad$ Experimental Results}

We have experimented with 3 syntheticly generated sets of trees with the properties given in 'lat ble 1 . In this tiable, the third column (label $\Lambda L^{3}$ ) gives the average ratio of the vertices at each level which are randomly selected as leaf vortices in a bec. 'The fourth columm gives the maximum number of children that a non-leaf node may have. 'The last column gives the maximum depth of the trees in that database.

from these synthetic databases, wo randomly extracted 100 trees and then perturbed them with random leaf deletions, insertions and label changes so that they were of some distance from it 


\begin{tabular}{|c|r|r|r|r|}
\hline Database & $\begin{array}{c}\text { Number } \\
\text { of } \\
\text { Trees }\end{array}$ & ALP & $\begin{array}{c}\text { Max } \\
\text { Children }\end{array}$ & $\begin{array}{c}\text { Max } \\
\text { Depth }\end{array}$ \\
\hline 1 & 1,000 & $1 / 3$ & 8 & 5 \\
2 & 10,000 & $1 / 2$ & 16 & 5 \\
3 & 50,000 & $1 / 2$ & 8 & 3 \\
\hline
\end{tabular}

Table 1: Properties of the synthetic databases of troos

tree in the original tree. We used thresholds $t=2$ and $t=4$, allowing an error of $C=1$ for cach leaf label change and an error of $S=2$ for each insertion or deletion (see Section 2.2). We then ran our algorithm on these data sets and obtained performance information. All runs were performed on a Sun SparcStation $20 / 61$ with $128 \mathrm{M}$ real memory. The rosults are presented in Table 2. It can be

\begin{tabular}{|c|c|c|r|r|}
\hline $\begin{array}{c}\text { Data- } \\
\text { base }\end{array}$ & $\begin{array}{c}\text { Thres- } \\
\text { hold }\end{array}$ & $\begin{array}{c}\text { Avg. } \\
\text { Leaves/ } \\
\text { Query } \\
\text { Irce }\end{array}$ & $\begin{array}{c}\text { Avg. } \\
\text { Search } \\
\text { Time } \\
\text { (Msec) }\end{array}$ & $\begin{array}{c}\text { Avg. } \\
\text { Irees } \\
\text { lound/ } \\
\text { Query }\end{array}$ \\
\hline 1 & 2 & 12.00 & 65 & 1.96 \\
& 4 & 12.42 & 81 & 16.65 \\
\hline 2 & 2 & 24.65 & 990 & 3.32 \\
& 4 & 25.62 & 1,659 & 31.59 \\
\hline 3 & 2 & 10.45 & 2,550 & 13.63 \\
& 4 & 10.45 & 3,192 & 68.62 \\
\hline
\end{tabular}

'lable 2: Performance results for the approximate trec matching algorithm.

secn that the approximate search algorithm is very fast for the set of synthetic tree databases that we have experimented with. It cortainly is also possible that additional space savings can be achieved if directed acyclic graphs can be used to represent the trec database taking into account both common prefixes and common suffixes of vertex list. sequences.

\section{Conclusions}

'I'his paper has presented an algorithm for approximate associative tree matching that can be used in example-based machine translation applications. 'The algorithm cfficiently searches in a database of trees, all trees that are "close" to a given query trce. The algorithm has been implemented on Sun Sparcstations, and experiments on rather largo synthetic tree database indicato that it can perform approximate matches within tenths of a second to few seconds depending on the size of the database and the error that the search is allowed to consider.

\section{Acknowledgments}

'This research was in part funded by a NATO Science for Stability Phase III Project Grant - TULANGUAGE.

\section{References}

M.W. Du and S. C. Chang. 1992. A model and a fast algorithm for multiple errors spelling correction. Acta Informatica, 29:281-302.

Sergei Nirenburg, Stephen Beale, and Constantine Domashnev. 1994. A Full-text Experiment in Fxample-based Translation. In Proceedings of the International Conference on New Methods in Language Processing, Manchester, UK, Pages $78-87$.

Kcmal Oflazer. 1996. Frror-tolcrant linite-state Recognition with $\Lambda$ pplications to Morphological Analysis and Spelling Correction, Computational Linguistics, Vol:22, No:1.

Satoshi Sato and Malkoto Nagao. 1990. Towards Memory-based 'Translation. In Proceedings of COLING'90 Vol.3, l'ages 247 -- 252.

Jason 'Tsong-Li Wang, Kaizhong 'thang, Karpjoo Jeong, and Dennis Shasha. 1994. $\Lambda$ System for $\Lambda$ pproximate Tree Matching. In IELIT Transactions of Knowledge and Data Engineering Vol. 6, No. 4, August, Pages $559-570$. 